\title{
CARACTERIZAÇÃO FÍSICO-QUÍMICA DE POLPAS DE FRUTOS DA AMAZÔNIA E SUA CORRELAÇÃO COM A ATIVIDADE ANTI-RADICAL LIVRE ${ }^{1}$
}

\author{
GISELE ANDRÉ BAPTISTA CANUTO², ANA AUGUSTA ODORISSI XAVIER ${ }^{3}$, \\ LEANDRO CAMARGO NEVES ${ }^{4}$, MARTA DE TOLEDO BENASSI ${ }^{5}$
}

RESUMO - Características físico-químicas (cor, pH, acidez total titulável, sólidos solúveis totais, conteúdo de lipídios e umidade) e níveis de compostos bioativos (ácido ascórbico, fenólicos totais) foram determinados em quinze amostras de polpas de frutos procedentes da região Amazônica (abiu, acerola, açaí, araçá-boi, bacaba, bacuri, buriti, cajá, cajarana, caju, cupuaçu, graviola, murici, noni e tamarindo). A atividade de radicais livres foi avaliada pelo método de ABTS. Algumas polpas apresentaram alta potencialidade antioxidante, associada com a atividade antirradicais livres obtida e os conteúdos dos componentes bioativos como compostos fenólicos e ácido ascórbico, destacando-se acerola e acaí. O conteúdo total de compostos fenólicos foi correlacionado à capacidade antioxidante das polpas.

Termos para indexação: ácido ascórbico, compostos fenólicos, TEAC, Análise de Componentes Principais.

\section{PHYSICAL AND CHEMICAL CHARACTERIZATION OF FRUIT PULPS FROM AMAZONIA AND THEIR CORRELATION TO FREE RADICAL SCAVENGER ACTIVITY}

\begin{abstract}
Physical and chemical characteristics (color, $\mathrm{pH}$, titratable acidity, total soluble solids, lipid content, moisture) and levels of bioactive compounds (ascorbic acid, total phenolics) were determined in fifteen samples of fruit pulps from Amazonia (abiu, acerola, açaí, araça-boi, bacaba, bacuri, buriti, cajá, cajarana, caju, cupuaçu, graviola, murici, noni e tamarindo). The free radical scavenger activity was evaluated by the ABTS assay. Some pulps presented high antioxidant potential, associated with the free radical scavenger activity measured and the content of bioactive components, such as phenolic compounds and ascorbic acid, especially in acerola and açaí. The total phenolic content was correlated to antioxidant capacity of pulps. Index terms: ascorbic acid, phenolic compounds, TEAC, Principal Component Analysis.
\end{abstract}

\section{INTRODUÇÃO}

O Brasil é o terceiro maior produtor de frutos (6\% da produção mundial) (ANDRIGUETO et al., 2010), mas a fruticultura Amazônica representa menos do que $0,2 \%$ desse total (ROMERO, 2009). A agricultura tradicional da região é composta basicamente por hortaliças, raízes nativas, plantas medicinais e frutos exóticos (CLAY et al., 1999), que são utilizadas tanto para consumo in natura quanto para elaboração de produtos processados (ANDRIGUETO et al., 2010; SOUSA et al., 2001). Nos últimos anos, houve um incremento da exploração econômica de produtos e subprodutos de algumas frutíferas específicas, atribuído à crescente preocupação do consumidor com a relação entre dieta e saúde (YAHIA, 2010). A caracterização física e química das frutos e a quantificação de componentes bioativos são importantes para o conhecimento do valor nutricional, e do ponto de vista comercial, para agregar valor e qualidade ao produto final. Dentre os compostos com propriedades funcionais em alimentos, substâncias com atividade antioxidante têm recebido grande atenção, pois auxiliam a proteger o organismo humano contra o estresse oxidativo, evitando e prevenindo uma série de distúrbios crônicodegenerativos (YAHIA, 2010).

Durante o processamento e estocagem, reações podem alterar as características originais dos alimentos, destacando-se as de autoxidação (LABUZA, 1971). A autoxidação é catalisada por fatores extrínsecos, inicia-se com a formação de radicais

\footnotetext{
'( Trabalho 214-09). Recebido em: 09-09-2009. Aceito para publicação em: 27-04-2010. Auxílio financeiro: CNPq e CAPES. ${ }^{2}$ Química. Mestranda no Instituto de Química, USP, 05508-000, São Paulo-SP, Brasil. gicanuto@usp.br

${ }^{3}$ Farm. Bioq. Ms. Doutoranda no Depto. de Ciência de Alimentos, FEA, UNICAMP, 13083-862, Campinas-SP, Brasil. anaaugustax@hotmail.com ${ }^{4}$ Eng. Agrônomo Dr. Professor, UFRR- Dept ${ }^{\circ}$. de Fitotecnia, 69301-970, Boa Vista-RR, Brasil. rapelbtu@hotmail.com ${ }^{5}$ Eng. Alimentos Dr. Professora Depto. de Ciência e Tecnologia de Alimentos, CCA, UEL, 86051-970, Londrina-PR, Brasil. martatb@uel.br
} 
livres e formam-se produtos extremamente reativos, que afetam atributos sensoriais, desenvolvendo cor, odor e sabor desagradáveis (HALLIWELL, 1996).

Para a inibição da produção de radicais livres, utilizam-se antioxidantes, substâncias que, presentes em baixas concentrações, se comparadas ao substrato, retardam significativamente ou inibem a oxidação do mesmo. Os radicais formados a partir de antioxidantes não são reativos para propagarem reações em cadeia (SOUSA et al., 2007). Entre os produtos naturais com atividade antirradical livre, encontram-se ácido ascórbico e numerosos compostos fenólicos (ARAÚJO, 1995; FENNEMA, 1996; SOARES, 2002; KITTS, 1994).

Já é reconhecida a relação entre ingestão de frutos e vegetais, e a diminuição do risco de desenvolvimento de diversas doenças crônicodegenerativas mediadas pela ação de radicais livres. Esses alimentos contêm grande concentração de compostos bioativos que possuem como função fisiológica, a ação contra radicais livres (AVELLO; SUWALSKY, 2006).

Diferentes métodos são utilizados para caracterizar a capacidade antioxidante de alimentos; entretanto, nenhum é universal (PRIOR et al., 2005). $\mathrm{O}$ método TEAC (Trolox Equivalent Antioxidant Capacity / Capacidade Antioxidante Equivalente ao Trolox) avalia a capacidade da amostra de absorver o radical 2,2'-azinobis(3-etilbenzotiazolina-6-ácido sulfônico $\left(\mathrm{ABTS}^{++}\right.$. É um método rápido, de fácil execução e pode ser correlacionado à atividade biológica, sendo um dos mais aplicados para realização de medidas da atividade antirradical livre de frutos, vegetais e seus produtos derivados (BERG et al., 2000; CÄMMERER; KROH, 2006; HUANG et al., 2005).

A caracterização de parâmetros para controle de qualidade $(\mathrm{pH}$, acidez total titulável, sólidos solúveis totais e cor), bem como de compostos com interesse funcional e atividade antirradical livre para polpas das espécies frutíferas amazônicas, permite valorizar esses produtos como alimento funcional e oferece opção de atividade sustentável para a população dessas regiões. $\mathrm{O}$ trabalho teve como objetivo a caracterização físico-química de quinze polpas processadas a partir de frutos colhidas na região da Amazônia, avaliando ainda parâmetros relacionados com possível atividade antirradical livre.

\section{MATERIAL E MÉTODOS}

\section{Preparação das polpas de frutos}

Os frutos utilizados foram: abiu (Pouteria caimito), acerola (Malpighia emarginata), açaí (Euterpe oleracea), araçá-boi (Eugenia stipitata), bacaba (Oenocarpus bacaba), bacuri (Platonia insignis), buriti (Mauritia flexuosa), cajá (Spondias lutea), cajarana (Spondias lutea Linn), caju (Anacardium occidentale), cupuaçu (Theobroma grandiflorum), graviola (Anona muricata), murici (Byrsonima crassifolia), noni (Morinda citrifolia) e tamarindo (Tamarindus indica).

Foram colhidos frutos no estádio de vez (fisiologicamente maturos, mature green state) provenientes de oito propriedades comerciais e cultivos espontâneos na Floresta Amazônica, na zona rural dos Estados de Roraima, Amazonas e Pará. Foram procedentes de Roraima: abiu, acerola e araçá-boi (região metropolitana de Boa Vista); bacaba, bacuri, murici e noni (região Centro-Sul do Estado); açaí (região Sul do estado) e caju (região Norte). Cupuaçu e graviolas foram procedentes do Estado do Pará (Belém) e cajá e cajarana do Amazonas (Manaus)

A coleta foi feita levando em consideração a padronização em relação ao tamanho e forma de cada espécie e quanto à ausência de danos mecânicos e contaminações aparentes e visuais na epiderme dos frutos. Foram selecionados para retirada de frutos com presença de injúrias e/ou deteriorações. Após seleção, foi realizada higienização em solução de hipoclorito $(50 \mathrm{ppm})$, previamente acidificada $(\mathrm{pH}$ $=3,0)$, por 10 minutos, e enxágue em bandejas perfuradas, com condições controladas de temperatura e umidade relativa $\left(21^{\circ} \mathrm{C}\right.$ e $\left.70 \%\right)$. Para a obtenção das polpas, foram retiradas cascas, epidermes, sementes e caroços das frutos, que posteriormente foram liquidificadas e congeladas.

\section{Análises Físico-Químicas}

Os teores de sólidos solúveis totais, $\mathrm{pH}$, acidez total titulável, umidade e lipídios foram determinados seguindo as Instituto Adolf Lutz (2008).

\section{Determinação de Cor}

Foi realizada utilizando colorímetro Chroma Meter CR-400 (Konica Minolta, Japão), empregando iluminante D65 e geometria 45/0, e os valores de cor foram expressos no sistema CIELAB. Os valores de $\mathrm{L}^{*}$ (luminosidade), a* (componente vermelho-verde) e $b^{*}$ (componente amarelo-azul) foram obtidos diretamente do colorímetro e utilizados para cálculo da tonalidade cromática $\left(\mathrm{H}^{*}=\arctan \mathrm{b}^{*} / \mathrm{a}^{*}\right)$ e croma $\left(C^{*}=\left(a^{* 2}+b^{* 2}\right)^{1 / 2}\right) . L^{*}$ varia de 0 a 100 , em que o 
valor 0 indica o preto (ou cor escura) e o 100, o branco (cor clara). Para $\mathrm{H}^{*}$, o 0 representa vermelho puro; o 90, o amarelo puro; o 180, o verde puro; e o 270, o azul puro. Assim, valores de $\left|\mathrm{H}^{*}\right|$ próximos de 90 , indicam tonalidade amarela, e, quanto mais próximos de 0 , a tonalidade vermelha. Com relação ao croma, quanto mais altos os valores de $\mathrm{C}^{*}$, mais viva a cor observada (LAWLESS; HEYMANN, 1998).

\section{Teor de Ácido Ascórbico (AA)}

As polpas $(25,0 \mathrm{~g})$ foram homogeneizadas em mixer, juntamente com 50,0 g de solução de ácido oxálico $2 \%(\mathrm{~m} / \mathrm{v})$. Foram retirados $20,0 \mathrm{~g}$ deste extrato, e o volume foi completado para $50,0 \mathrm{~mL}$, em balão volumétrico, com a solução de ácido oxálico a $2 \%(\mathrm{~m} / \mathrm{v})$. Utilizou-se uma alíquota de $10,0 \mathrm{~mL}$ desta solução do balão para titulação com 2,6-diclorofenolindofenol $0,01 \%(\mathrm{~m} / \mathrm{v})$, previamente padronizado com solução-padrão de ácido ascórbico (BENASSI; ANTUNES, 1988).

\section{Teor de Fenóis Totais (FT)}

Para a extração dos compostos fenólicos, foram adicionados $50,0 \mathrm{~mL}$ de solução metanol:água $(8: 2)(\mathrm{v} / \mathrm{v})$ a $10,0 \mathrm{~g}$ de polpa, seguido de agitação em ultrassom por 20 minutos. A mistura foi filtrada e lavada com $20 \mathrm{~mL}$ de metanol, e transferiu-se o filtrado para um balão de $100,0 \mathrm{~mL}$, e o volume foi completado com solução metanol:água (8:2). Após 1 hora em freezer $\left(-20^{\circ} \mathrm{C}\right)$, a solução foi centrifugada a $2.000 \mathrm{rpm}$,durante 20 minutos, e o sobrenadante foi retirado e acondicionado em freezer. Para a determinação de teor de fenóis totais, uma alíquota de $100 \mu \mathrm{L}$ de amostra ou padrão foi colocada em tubo de ensaio, com 7,5 mL de água ultrapura e $0,30 \mathrm{~mL}$ de reagente de Folin-Ciocalteau 0,9 mol.L ${ }^{-1}$. Após agitação, foram adicionados $1,0 \mathrm{~mL}$ de solução de carbonato de sódio a $20 \%(\mathrm{~m} / \mathrm{v})$ e $1,1 \mathrm{~mL}$ de água destilada. Os reagentes foram misturados, e os tubos foram deixados em repouso a $25^{\circ} \mathrm{C}$ por 1 hora. Construiu-se curva de calibração com 8 concentrações $(0,05 ; 0,10$; 0,$25 ; 0,5 ; 1,0 ; 2,0$ e 5,0 mmol. $\left.\mathrm{L}^{-1}\right)$ de ácido gálico, em triplicata. A medida de absorvância foi realizada em espectrofotômetro a $765 \mathrm{~nm}$ (KIM et al., 2003).

\section{Atividade Antirradical livre (TEAC)}

Empregou-se o mesmo procedimento de extração utilizado na determinação de polifenóis totais. $\mathrm{O}$ cátion $\mathrm{ABTS}^{\cdot+}$ foi produzido pela reação de 7,0 mmol.L $\mathrm{L}^{-1}$ de uma solução-estoque de ABTS com 2,5 mmol. $\mathrm{L}^{-1}$ de persulfato de potássio. A mistura foi armazenada em frasco escuro, em temperatura ambiente, por 12-16 horas antes do uso. A solução $\mathrm{ABTS}^{\circ+}$ foi então diluída com tampão fosfato $(\mathrm{pH} 7,4)$ até absorvância de 0,7 $\pm 0,01$ a $730 \mathrm{~nm}$. Em um tubo de reação, foram adicionados $10,0 \mu \mathrm{L}$ de amostra ou padrão Trolox ${ }^{\circledR}$ e 4,0 mL da solução $\mathrm{ABTS}^{++}$diluída. Após seis minutos de reação, fez-se a leitura em espectrofotômetro a 730 nṃutilizando água ultrapura como branco. A curva de calibração foi feita utilizando soluções etanólicas de Trolox nas seguintes concentrações: 1,$0 ; 2,0 ; 3,0 ; 5,0$ e 8,0 mmol. $\mathrm{L}^{-1}$. A atividade equivalente ao Trolox (TEAC) foi dada pela porcentagem de inibição ${ }^{\circledR}$ do radical ABTS (\%IA) (Equação 1) (SÁNCHEZ-GONZÁLEZ et al., 2005).

$\%$ IA $=100-($ Abs Amostra/Abs Controle $) \times 100$

Equação (1)

\section{Análise Estatística}

Empregou-se um delineamento inteiramente ao acaso. Os resultados de cada parâmetro foram submetidos à análise de variância (ANOVA) e teste de Tukey (5\% de significância). Para correlação entre a atividade antirradical livre e os teores de fenóis totais e ácido ascórbico, foi empregada regressão multilinear. A caracterização das polpas pelos constituintes químicos e atividade antirradical foi realizada pela análise de componentes principais (ACP) e análise de agrupamentos. O dendrograma foi obtido por algoritmo de agrupamento não ponderado aos pares (UPGMA), considerando-se a distância euclidiana como coeficiente de semelhança. Em todas as análises, foi utilizado o software Statistica 6.0 (STATSOFT, 2001).

\section{RESULTADOS E DISCUSSÃO}

A maioria das polpas estudadas (noni, murici, abiu, cajá, cajarana, caju, bacuri, cupuaçu e graviola) apresentou cor amarelada indicada por valor de tonalidade cromática alto $\left(\left|\mathrm{H}^{*}\right|\right.$ próximo ou acima de 70). A tonalidade de amarelo variou de amarelo ligeiramente esverdeado (valores negativos de $\mathrm{H}^{*}$ ) até um pouco alaranjado (valores positivos de $\mathrm{H}^{*}$ ). Açaí, araçá-boi, bacaba e tamarindo caracterizaramse por uma tonalidade amarelo-avermelhada $\left(\mathrm{H}^{*} \mathrm{de}\right.$ 63 a 49), e acerola mostrou uma tonalidade avermelhada $\left(\mathrm{H}^{*}\right.$ em torno de 20$)$. Com relação ao croma $\left(C^{*}\right)$, observaram-se valores variando de 3,2 até 42,9 (graviola e buriti, respectivamente), indicando que a graviola apresenta uma coloração mais opaca, enquanto o buriti mostra uma cor mais viva. $\mathrm{O}$ açaí foi a polpa de cor mais escura $\left(\mathrm{L}^{*}=16,6\right)$, e a de bacuri, a mais clara $\left(\mathrm{L}^{*}=65,5\right)$ (Tabela 1$)$.

A faixa de umidade encontrada para as polpas foi 75 a $97 \%$ (Tabela 2). 
Bacaba $(7,4 \%)$ e açaí $(4,6 \%)$ foram as polpas que apresentaram maiores teores de lipídios. Bacuri e buriti mostraram valores na faixa de $1,0 \%$, e para todas as outras polpas, observou-se concentração inferior a 0,6 \% (Tabela 2).

O teor de sólidos solúveis totais apresenta correlação com teores açúcares e ácidos orgânicos, característica de interesse para produtos comercializados in natura, pois o mercado consumidor prefere frutos doces (SILVA et al., 2002). Dentre as polpas analisadas, o tamarindo foi a que apresentou maior teor de sólidos solúveis totais $\left(24,0{ }^{\circ} \mathrm{Brix}\right)$, provavelmente devido não só aos açucares, mais ao alto teor de acidez total titulável encontrado: 30,59 mg de ácido cítrico/ $100 \mathrm{~g}$ de amostra (Tabela 2). Observou-se que, para os produtos estudados, as polpas com alto teor de sólidos solúveis totais, como açaí, bacuri, graviola, cupuaçu e noni (de 18,0 a 9,0 ${ }^{\circ}$ Brix), apresentaram menores teores de umidade. A polpa de murici apresentou o menor teor de sólidos solúveis totais $\left(1,5^{\circ} \mathrm{Brix}\right)$.

A faixa de $\mathrm{pH}$ variou de 2,5 a 5,3 (tamarindo e bacaba, respectivamente), e os teores de acidez total titulável encontrados foram de $0,1 \mathrm{mg}$ de ácido cítrico/100 g na bacaba a 45,9 mg de ácido cítrico/100 g de polpa no açaí (Tabela 2).

Alves et al. (2000) descrevem a composição de frutos nativos da América Latina, entre as quais açaí, araçá-boi, bacuri, cajá e cupuaçú. Apesar da dificuldade para comparação, uma vez que neste trabalho os frutos foram processados para polpa e congeladas o que poderia implicar modificação na composição, observou-se similaridade em alguns dados. Para bacuri, cajá e cupuaçu, forma descritos valores de $\mathrm{pH}$ bastante próximos $(3,37 ; 3,17$ e 3,30) aos observados no trabalho (Tabela 2). Os autores relataram valor mais baixo para o $\mathrm{pH}$ de araçá-boi $(2,50)$ e mais alto para açaí $(5,50)$. Quanto ao teor de sólidos solúveis totais, observaram-se valores próximos ao obtido no trabalho (Tabela 2) para araçáboi e bacuri (5,2 e 13,0 ${ }^{\circ}$ Brix, respectivamente). Os autores relataram menor teor de sólidos totais para açaí $\left(7,55^{\circ}\right.$ Brix $)$ e maior teor para cajá e cupuaçu $\left(11,56\right.$ e $16,37^{\circ}$ Brix $)$

A polpa de acerola apresentou o maior teor de ácido ascórbico $(378,5 \mathrm{mg} / 100 \mathrm{~g})$ (Tabela 3), valor, no entanto, inferior a alguns dos citados na literatura para o fruto, na faixa de 514,00 a 1.655,00 $\mathrm{mg} / 100 \mathrm{~g}$ (OLIVEIRA et al., 1999). O teor deste composto pode variar entre diferentes regiões do País, em função de fatores como temperatura, intensidade de luz e conteúdo de umidade, além do processamento da polpa, que pode afetar bastante a concentração de ácido ascórbico (RUFINO et al.,
2009). Rufino et al. (2009) descrevem teores altos de ácido ascórbico para açaí, caju e murici (84; 190 e $148 \mathrm{mg} / 100 \mathrm{~g}$, respectivamente), enquanto para as polpas desses produtos, valores muito inferiores foram observados $(10 ; 12$ e $0,3 \mathrm{mg} / 100 \mathrm{~g})$. Barreto et al. (2009) relataram teores de ácido ascórbico para polpas de bacuri, buriti a murici inferiores a $1 \mathrm{mg} / 100 \mathrm{~g}$ $(0,5 ; 0,7$ e $0,4 \mathrm{mg} / 100 \mathrm{~g})$, e próximos aos observados no trabalho (Tabela 3 ).

A Tabela 3 apresenta, também, a concentração de compostos fenólicos, podendo-se observar que as polpas de acerola e açaí se destacaram ainda pelo elevado teor de fenóis totais $\left(3,45\right.$ e 2,4 mmol. $\mathrm{L}^{-1}$ de ácido gálico/ $100 \mathrm{~g}$ de amostra). Alves et al. (2000) relataram teores de fenólicos solúveis em $50 \%$ de metanol, em torno de 10 vezes superiores para o fruto de açaí comparado com araçá-boi, bacuri, cajá e cupuaçu. Rufino et al. (2009) apresentam para acerola, açaí, caju e murici, valores de 6,24; 3,04; 0,70 e 5,51 mmol. $\mathrm{L}^{-1}$ de ácido gálico/100 $\mathrm{g}$ de fruto, respectivamente. A concentração de compostos fenólicos para acerola e murici foi muito superior à observada neste trabalho (Tabela 3). Barreto et al. (2009) relataram teor de fenóis totais de 1,56; 0,63 e 2,26 mmol.L-1 de ácido gálico/100 g para polpas de bacuri, buriti e murici, respectivamente. $\mathrm{O}$ teor para buriti foi próximo ao observado no trabalho $\left(1,0 \mathrm{mmol} . \mathrm{L}^{-1}\right.$ de ácido gálico/100 g), mas observou-se valores em torno de quatro vezes superiores de fenóis para bacuri e murici do que os obtidos neste estudo $\left(0,4\right.$ e 0,6 mmol. $\mathrm{L}^{-1}$ de ácido gálico/100 g, respectivamente) (Tabela 3). Essa discrepância pode ser atribuída a fatores edafoclimáticos e/ou genéticos mas é provável que a diversidade no estádio de maturidade dos frutos empregados no preparo das polpas seja a maior responsável pela variabilidade.

Em relação à atividade antirradical livre, novamente as polpas de açaí e acerola apresentaram os maiores valores $\left(12,1 \mathrm{e} 10,0 \mu \mathrm{mol}\right.$ Trolox. $\mathrm{L}^{-1}$, respectivamente), indicando provável relação entre os níveis de ácido ascórbico e fenóis totais e o potencial antioxidante do fruto. Abiu, bacuri e cupuaçu foram as polpas que demonstraram a menor atividade antirradical livre, inferior à $0,8 \mu \mathrm{mol} . \mathrm{L}^{-1}$. Barreto et al. (2009) relataram valores de TEAC de 5,4; 16,4 e $18,4 \mu \mathrm{mol} . \mathrm{L}^{-1}$ para polpas de buriti, bacuri e murici, respectivamente. Observou-se também, nesse parâmetro, concordância para a atividade antirradical livre de buriti, mas valores muito superiores para bacuri e murici do que os obtidos neste trabalho $(0,6$ e $1,5 \mu \mathrm{mol} . \mathrm{L}^{-1}$, respectivamente). Considerando-se a diferença nos teores de fenóis totais já discutidos anteriormente, a diversidade na atividade antirradical livre seria esperada. 
Através de uma análise de regressão multilinear, observou-se que as duas variáveis (teor de ácido ascórbico e fenóis totais) podem ser correlacionadas à atividade antirradical livre $\left(\mathrm{R}^{2}=0,93, \mathrm{p}<0,001\right)$ (Equação 2).

$$
\begin{aligned}
& \text { TEAC }=-0,007 \text { AA + 4,34 FT } \quad \text { Equação (2) } \\
& \left(R^{2}=0,93 ; p<0,001\right) .
\end{aligned}
$$

Os fenóis apresentaram a maior contribuição para a atividade antioxidante das polpas. Apesar dos elevados teores de ácido ascórbico constatados em algumas polpas, esse composto não teve contribuição na explicação da atividade antirradical livre, fato observado também na análise de componentes principais (Figura 1). Assim, pode-se afirmar que, para as polpas analisadas, existe boa correlação entre os valores de TEAC e concentração de compostos fenólicos, e que somente a determinação do teor de fenólicos totais já seria um bom indicativo da atividade antirradical livre das mesmas.

$\mathrm{Na}$ literatura, muitos autores relatam correlação entre a atividade antirradical livre medida por ABTS e fenólicos totais (CHUN et al., 2003; SILVA et al., 2007; CAI et al., 2004). Para o ácido ascórbico, apesar do seu reconhecido potencial antioxidante, não existe o mesmo consenso. Os trabalhos de Gil et al. (2002) e Luximon-Ramma et al. (2003) relatam que não se observou correlação entre a atividade antiradical livre e os níveis de ácido ascórbico. Barreto et al. (2009) estudaram 18 polpas de frutos tropicais e observaram que a atividade antirrradical livre encontrada mostrou alta correlação com teor de compostos fenólicos totais e flavonoides; mas a atividade antirradical livre não foi dependente da concentração de ácido ascórbico e carotenoides totais.

Os dois primeiros componentes permitiram uma explicação de 67 \%. Na Figura 1, podem ser observados a projeção das variáveis (A) e o gráfico de amostras (B), com a dispersão dos pontos. No dendrograma, pode ser observada a separação entre os grupos através do corte realizado (linha pontilhada) (Figura 2), utilizado para separação dos grupos na Figura 1.

O CP1 foi correlacionado com atributos de cor e atividade antirradical livre. Foi associado positivamente a $L^{*}$ e negativamente ao valor de $a^{*}$, fenóis totais, ácido ascórbico e TEAC. Polpas alocadas à direita caracterizaram-se como mais claras, amareladas e com menor potencial antirradical livre. O CP2 pode ser caracterizado principalmente por parâmetros físico-químicos, como sólidos solúveis totais e acidez total titulável (positivamente) e umidade (negativamente). Polpas alocadas na parte superior do gráfico de amostras caracterizaram-se por apresentar maior acidez total titulável e teor de sólidos solúveis totais (Figura 1 A e B).A Figura 1A permite-nos ainda observar a correlação negativa entre os parâmetros de lipídios e umidade, e entre acidez e $\mathrm{pH}$, bem como a correlação positiva entre o teor de ácido ascórbico e compostos fenólicos totais e TEAC.

Quatro polpas (açaí, tamarindo, acerola e bacaba) foram separadas cada uma como um grupo, por apresentarem características que as diferenciam das demais (Figuras 1 e 2).Acerola e açaí destacaramse pelo alto potencial antirradical livre. $\mathrm{O}$ açaí foi caracterizado pelo alto teor de SS e coloração mais escura. A acerola apresentou o maior teor de vitamina $\mathrm{C}$, fenóis totais e, consequentemente, TEAC. Também possui maior teor de umidade e coloração alaranjada característica (Tabelas 1 e 2).O tamarindo apresentou cor escura e alto teor de SS e lipídios, sendo consequentemente a polpa com menor teor de umidade (Figura 1, Tabela 2). A bacaba foi separada na análise de agrupamentos (Figura 2), apesar de ser alocada muito próxima às demais polpas na ACP (Figura 1). Isso ocorreu principalmente devido ao alto teor de lipídios (4).

Os dois maiores grupos (5 e 6) foram separados pela presença de polpas com cor mais clara (Tabela 1). O grupo 5 é composto por graviola, bacuri, noni e cupuaçu, que são polpas de alta acidez (Tabela 2). Abiu, araçá-boi, buriti, cajá, cajarana, caju e murici (grupo 6) têm como características em comum o baixo teor de fenóis totais (menor do que 1,00 mmol.L $\mathrm{L}^{-1}$ de ácido gálico), baixa porcentagem de lipídios, e pela coloração amarelada (Tabelas 1 e 2). 
TABELA 1 - Caracterização das polpas de frutos da Amazônia quanto à cor.

\begin{tabular}{cccc}
\hline Amostra & $L^{*}$ & $H^{*}$ & $C^{*}$ \\
\hline Abiu & $46,2^{\mathrm{de}} \pm 0,4$ & $85,4^{\mathrm{a}} \pm 2,8$ & $26,2^{\mathrm{ef}} \pm 1,5$ \\
Acerola & $23,8^{\mathrm{g}} \pm 0,3$ & $19,6^{\mathrm{f}} \pm 1,4$ & $33,2^{\mathrm{h}} \pm 0,1$ \\
Açaí & $16,6^{\mathrm{h}} \pm 1,6$ & $48,7^{\mathrm{g}} \pm 0,1$ & $12,9^{\mathrm{cd}} \pm 1,1$ \\
Araçá-boi & $40,7^{\mathrm{ef}} \pm 0,3$ & $49,5^{\mathrm{ef}} \pm 0,2$ & $29,8^{\mathrm{de}} \pm 2,6$ \\
Bacaba & $26,8^{\mathrm{g}} \pm 0,4$ & $56,0^{\mathrm{de}} \pm 0,1$ & $20,0^{\mathrm{g}} \pm 1,0$ \\
Bacuri & $65,5^{\mathrm{a}} \pm 2,1$ & $-75,6^{\mathrm{i}} \pm 0,2$ & $9,5^{\mathrm{h}} \pm 1,3$ \\
Buriti & $48,2^{\mathrm{cd}} \pm 3,0$ & $70,8^{\mathrm{c}} \pm 0,8$ & $42,9^{\mathrm{a}} \pm 1,3$ \\
Cajá & $47,9^{\mathrm{d}} \pm 3,1$ & $73,7^{\mathrm{bc}} \pm 0,6$ & $32,7^{\mathrm{cd}} \pm 0,4$ \\
Cajarana & $56,1^{\mathrm{b}} \pm 2,2$ & $79,6^{\mathrm{ab}} \pm 0,6$ & $36,5^{\mathrm{bc}} \pm 0,5$ \\
Caju & $57,6^{\mathrm{b}} \pm 0,5$ & $79,0^{\mathrm{ab}} \pm 0,2$ & $31,9^{\mathrm{cd}} \pm 0,6$ \\
Cupuaçu & $54,9^{\mathrm{bc}} \pm 3,1$ & $-77,8^{\mathrm{i}} \pm 0,0$ & $8,4^{\mathrm{h}} \pm 0,8$ \\
Graviola & $56,0^{\mathrm{bc}} \pm 1,8$ & $-66,2^{\mathrm{h}} \pm 3,0$ & $3,2^{\mathrm{i}} \pm 0,3$ \\
Murici & $45,8^{\mathrm{de}} \pm 1,0$ & $80,6^{\mathrm{ab}} \pm 1,2$ & $39,3^{\mathrm{ab}} \pm 0,3$ \\
Noni & $47,8^{\mathrm{d}} \pm 0,2$ & $86,0^{\mathrm{a}} \pm 5,2$ & $8,4^{\mathrm{h}} \pm 1,4$ \\
Tamarindo & $33,8^{\mathrm{f}} \pm 0,5$ & $63,1^{\mathrm{d}} \pm 0,2$ & $23,3^{\mathrm{fg}} \pm 1,6$ \\
\hline
\end{tabular}

Dados expressos como média de duplicata \pm desvio-padrão. Médias seguidas da mesma letra, nas colunas, não diferem pelo teste de Tukey $(\mathrm{p} \leq 0,01)$.

TABELA 2 - Valores de umidade, lipídios, sólidos solúveis totais, pH e acidez total titulável de polpas de frutos da Amazônia.

\begin{tabular}{|c|c|c|c|c|c|}
\hline Amostra & Umidade $^{l}$ & Lipídios $^{1}$ & $\begin{array}{c}\text { Sólidos Solúveis } \\
\text { Totais }^{2}\end{array}$ & $p H$ & $\begin{array}{l}\text { Acidez Total } \\
\text { Titulável }^{3}\end{array}$ \\
\hline Abiu & $95,8^{b} \pm 0,2$ & $0,1^{\mathrm{c}} \pm 0,0$ & $3,8^{\mathrm{fg}} \pm 0,4$ & $5,0^{\mathrm{ab}} \pm 0,2$ & $5,9^{\mathrm{c}} \pm 0,1$ \\
\hline Acerola & $97,5^{\mathrm{a}} \pm 0,0$ & $0,2^{\mathrm{c}} \pm 0,1$ & $3,5^{\mathrm{fg}} \pm 2,1$ & $2,8^{\mathrm{fgh}} \pm 0,4$ & $1,9^{\mathrm{g}} \pm 0,0$ \\
\hline Açaí & $83,3^{\mathrm{h}} \pm 0,2$ & $4,6^{\mathrm{b}} \pm 0,0$ & $18,0^{b} \pm 0,0$ & $3,3^{\mathrm{hi}} \pm 0,0$ & $45,9^{\mathrm{a}} \pm 0,0$ \\
\hline Araçá-boi & $90,1^{\mathrm{f}} \pm 0,5$ & $0,3^{\mathrm{c}} \pm 0,1$ & $4,5^{\mathrm{fg}} \pm 1,4$ & $4,0^{\text {cdef }} \pm 0,1$ & $1,8^{\mathrm{gh}} \pm 0,1$ \\
\hline Bacaba & $87,6^{\mathrm{g}} \pm 0,4$ & $7,4^{\mathrm{a}} \pm 1,8$ & $2,0^{\mathrm{g}} \pm 0,7$ & $5,3^{\mathrm{a}} \pm 0,1$ & $0,1^{1} \pm 0,0$ \\
\hline Bacuri & $84,8^{\mathrm{h}} \pm 0,8$ & $1,1^{\mathrm{c}} \pm 0,6$ & $13,0^{c} \pm 1,8$ & $3,4^{\mathrm{fgh}} \pm 0,2$ & $2,9^{\mathrm{e}} \pm 0,1$ \\
\hline Buriti & $89,9 \pm 0,7$ & $1,7^{\mathrm{c}} \pm 0,2$ & $4,5^{\mathrm{fg}} \pm 0,7$ & $3,5^{\mathrm{efg}} \pm 0,1$ & $2,2^{\mathrm{f}} \pm 0,0$ \\
\hline Cajá & $91,7^{\mathrm{de}} \pm 0,2$ & $0,2^{\mathrm{c}} \pm 0,1$ & $6,0^{\mathrm{ef}} \pm 0,7$ & $2,9^{\text {ghi }} \pm 0,4$ & $1,3^{\mathrm{ij}} \pm 0,1$ \\
\hline Cajarana & $96,1^{\mathrm{ab}} \pm 0,2$ & $0,1^{\mathrm{c}} \pm 0,0$ & $4,8^{\mathrm{fg}} \pm 0,4$ & $4,4^{\mathrm{bcd}} \pm 0,1$ & $0,4^{\mathrm{k}} \pm 0,0$ \\
\hline Caju & $93,8^{c} \pm 0,0$ & $0,1^{\mathrm{c}} \pm 0,1$ & $5,0^{\mathrm{fg}} \pm 0,7$ & $4,7^{\mathrm{abc}} \pm 0,0$ & $0,2^{\mathrm{kl}} \pm 0,0$ \\
\hline Cupuaçu & $89,2^{\mathrm{fg}} \pm 0,3$ & $0,3^{\mathrm{c}} \pm 0,0$ & $9,0^{\mathrm{de}} \pm 0,0$ & $3,5^{\text {efgh }} \pm 0,2$ & $3,5^{\mathrm{d}} \pm 0,0$ \\
\hline Graviola & $88,1^{\mathrm{g}} \pm 0,1$ & $0,1^{\mathrm{c}} \pm 0,0$ & $12,0^{\mathrm{cd}} \pm 0,0$ & $3,7^{\mathrm{def}} \pm 0,1$ & $1,5^{\mathrm{hi}} \pm 0,0$ \\
\hline Murici & $92,8^{\mathrm{cd}} \pm 0,3$ & $0,6^{\mathrm{c}} \pm 0,2$ & $1,5^{\mathrm{g}} \pm 0,0$ & $3,7^{\mathrm{def}} \pm 0,2$ & $1,0^{\mathrm{j}} \pm 0,0$ \\
\hline Noni & $90,2^{\mathrm{ef}} \pm 0,1$ & $0,1^{\mathrm{c}} \pm 0,0$ & $9,0^{\mathrm{de}} \pm 0,0$ & $4,1^{\text {cde }} \pm 0,2$ & $3,2^{\mathrm{e}} \pm 0,0$ \\
\hline Tamarindo & $75,0^{\mathrm{i}} \pm 0,8$ & $0,1^{\mathrm{c}} \pm 0,0$ & $24,0^{\mathrm{a}} \pm 0,0$ & $2,5^{\mathrm{i}} \pm 0,2$ & $30,6^{b} \pm 0,2$ \\
\hline
\end{tabular}

${ }^{1}$ expresso como g/100 g de polpa; ${ }^{2}$ expresso em ${ }^{\circ}$ Brix $;{ }^{3}$ expresso em $\mathrm{mg}$ ácido cítrico/100 g de polpa. Dados expressos como média de duplicata \pm desvio-padrão. Médias seguidas da mesma letra, nas colunas, não diferem pelo teste de Tukey $(\mathrm{p} \leq 0,01)$. 
TABELA 3 - Teores de ácido ascórbico, fenóis totais e atividade antirradical livre (TEAC) de polpas de frutos da Amazônia.

\begin{tabular}{cccc}
\hline Amostra & $A A^{1}$ & $F T^{2}$ & $T E A C^{3}$ \\
\hline Abiu & $2,0^{\mathrm{g}} \pm 0,0$ & $0,4^{\mathrm{fg}} \pm 0,0$ & $0,8^{\mathrm{f}} \pm 0,1$ \\
Acerola & $378,5^{\mathrm{a}} \pm 0,0$ & $3,5^{\mathrm{a}} \pm 0,2$ & $12,1^{\mathrm{a}} \pm 0,0$ \\
Açaí & $10,1^{\mathrm{e}} \pm 0,0$ & $2,4^{\mathrm{b}} \pm 0,2$ & $10,0^{\mathrm{b}} \pm 0,3$ \\
Araçá-boi & $0,2^{\mathrm{i}} \pm 0,0$ & $0,6^{\mathrm{ef}} \pm 0,0$ & $3,0^{\mathrm{de}} \pm 0,1$ \\
Bacaba & $0,9^{\mathrm{h}} \pm 0,2$ & $0,3^{\mathrm{g}} \pm 0,1$ & $3,1^{\mathrm{de}} \pm 2,1$ \\
Bacuri & $0,2^{\mathrm{i}} \pm 0,0$ & $0,4^{\mathrm{g}} \pm 0,0$ & $0,6^{\mathrm{f}} \pm 0,3$ \\
Buriti & $0,1^{\mathrm{i}} \pm 0,0$ & $1,0^{\mathrm{cd}} \pm 0,0$ & $5,4^{\mathrm{c}} \pm 0,2$ \\
Cajá & $0,3^{\mathrm{i}} \pm 0,0$ & $0,6^{\mathrm{ef}} \pm 0,0$ & $1,8^{\mathrm{ef}} \pm 0,4$ \\
Cajarana & $18,3^{\mathrm{c}} \pm 0,0$ & $0,4^{\mathrm{fg}} \pm 0,1$ & $1,4^{\mathrm{ef}} \pm 0,1$ \\
Caju & $12,4^{\mathrm{d}} \pm 0,0$ & $0,6^{\mathrm{ef}} \pm 0,0$ & $1,5^{\mathrm{ef}} \pm 0,2$ \\
Cupuaçu & $3,3^{\mathrm{f}} \pm 0,0$ & $0,4^{\mathrm{fg}} \pm 0,0$ & $0,6^{\mathrm{f}} \pm 0,2$ \\
Graviola & $0,1^{1} \pm 0,0$ & $0,6^{\mathrm{ef}} \pm 0,0$ & $2,2^{\mathrm{def}} \pm 0,1$ \\
Murici & $0,3^{\mathrm{i}} \pm 0,1$ & $0,6^{\mathrm{ef}} \pm 0,0$ & $1,5^{\mathrm{ef}} \pm 1,5$ \\
Noni & $51,2^{\mathrm{b}} \pm 0,0$ & $1,2^{\mathrm{c}} \pm 0,1$ & $3,9^{\mathrm{cd}} \pm 0,1$ \\
Tamarindo & $0,1^{\mathrm{i}} \pm 0,0$ & $0,8^{\mathrm{de}} \pm 0,1$ & $2,9^{\mathrm{de}} \pm 0,2$ \\
\hline
\end{tabular}

${ }^{1}$ Ácido Ascórbico, expresso em mg/100g de polpa; ${ }^{2}$ Fenóis Totais, expresso como mmol.L ${ }^{-1}$ de ácido gálico; ${ }^{3}$ Atividade Antirradical livre Equivalente ao Trolox, expresso como $\mu$ mol. $\mathrm{L}^{-1}$ de Trolox. Dados expressos como média de duplicata \pm desvio-padrão. Médias seguidas da mesma letra, nas colunas, não diferem pelo teste de Tukey $(\mathrm{p} \leq 0,01)$.
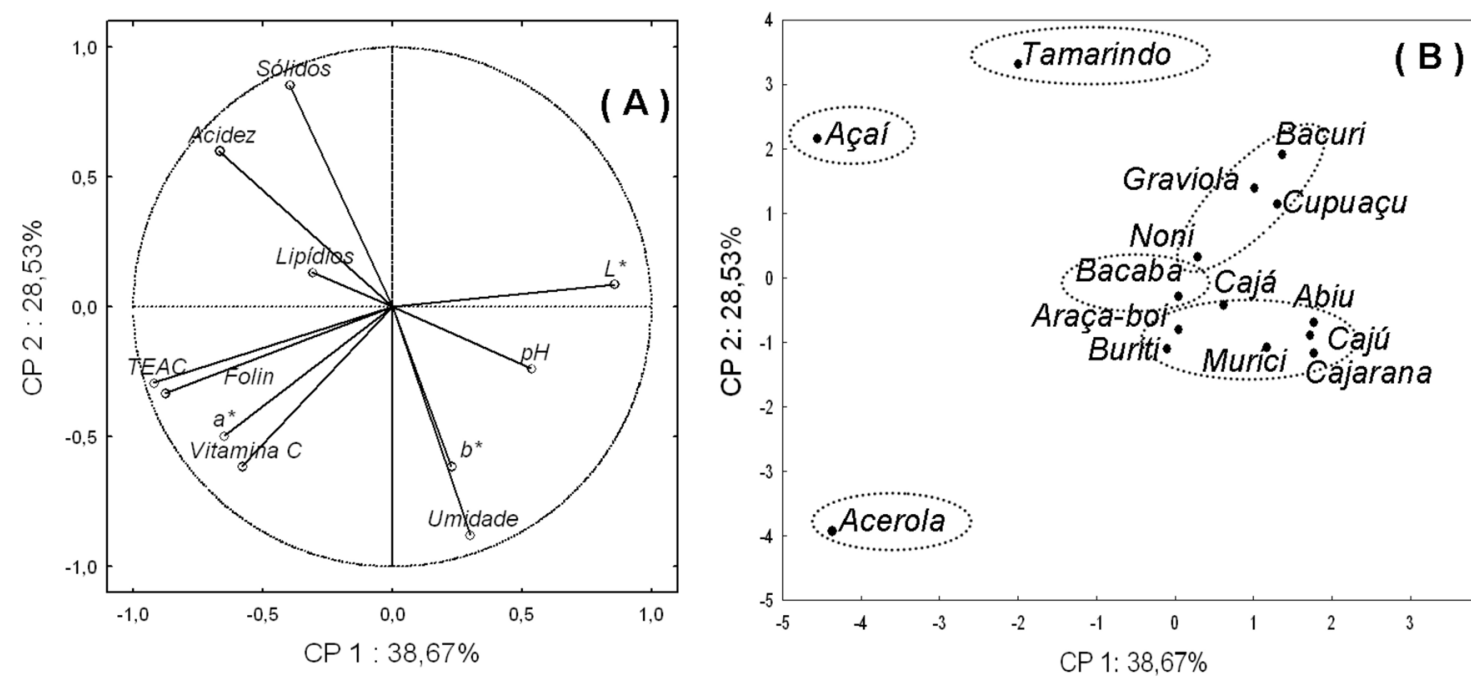

FIGURA 1 - Análise de Componentes Principais, utilizando os parâmetros físico-químicos e atividade antirradical livre de polpas de frutos da Amazônia: projeção das variáveis (A) e gráfico de amostras (B). 


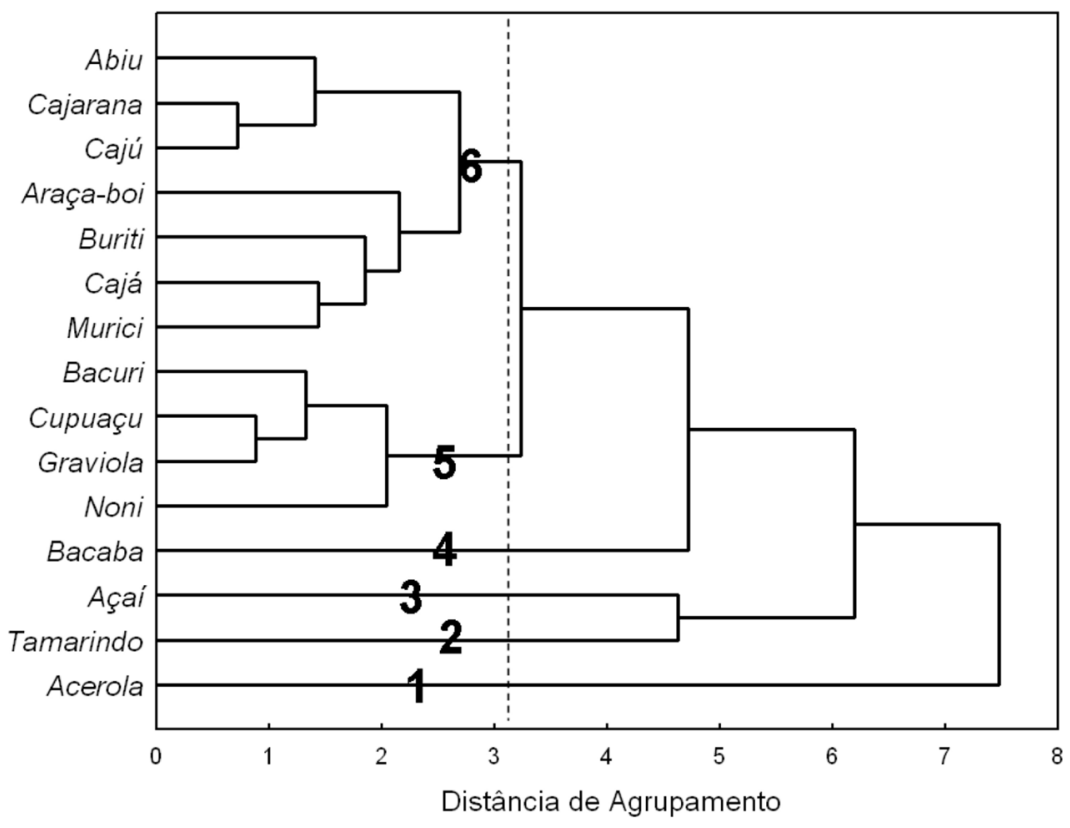

FIGURA 2 - Dendograma obtido a partir da caracterização físico-química e determinação da atividade antirradical livre de polpas de frutos da Amazônia.

\section{CONCLUSÕES}

A avaliação de parâmetros físico-químicos e da atividade antirradical livre das polpas de frutos tropicais da região Amazônica permite boa caracterização destas quanto aos compostos bioativos presentes, bem como a cor. Observa-se correlação entre atividade antirradical livre e teores de ácido ascórbico e, principalmente, compostos fenólicos totais. Várias polpas apresentam bom potencial antirradical livre, detectada tanto pela medida específica da atividade quanto pela inibição de radicais livres, bem como pela presença de compostos bioativos como fenóis e ácido ascórbico, destacando-se acerola e açaí.

\section{AGRADECIMENTOS}

Ao CNPq, pela bolsa de Iniciação Científica e pelo financiamento do projeto Pós-Colheita, Processamento e Comercialização de Frutos Nativos da Amazônia, Prroduzidos em Sistemas Agrofloretais Familiares, aprovado no edital 039/2007.

\section{REFERÊNCIAS}

ALVES, R.E.; FILGUEIRAS, H.A.C.; MOURA, C.F.H. Caracterização de frutas nativas da América Latina. Jaboticabal: Funep, 2000. 66 p. (Série Frutas Nativas, 9).

ANDRIGUETO, J.R.; NASSER, L.C.B.; TEIXEIRA, J.M.A. Produção integrada de frutas: conceito, histórico e a evolução para o sistema agropecuário de produção integrada -SAPI. Disponível em: <www. agricultura.gov.br>. Acesso em: mar. 2010.

ARAÚJO, J.M.A. Química dos alimentos: teoria e prática. Viçosa: UFV, 1995. 335 p.

AVELLO, M.; SUWALSKY, M. Radicales libres, antioxidantes naturales y mecanismos de proteccion. Atena, Concepción, n.494, n. 2, p. 161-172, 2006.

BARRETO, G.P.M., BENASSI, M.T.; MERCADANTE, A.Z. Bioactive compounds from several tropical fruits and correlation by multivariate analysis to free radical scavenger activity. Journal of Brazilian Chemical Society, São Paulo, v. 20, n. 10, p. 1856-1861, 2009. 
BENASSI, M.T.; ANTUNES, A.J. A comparison of metaphosphoric and oxalic acids as extractant solutions for the determination of vitamin $\mathrm{C}$ in selected vegetables. Arquivos de Biologia e Tecnologia, Curitiba, v. 31, n. 4, p. 507-513, 1988.

BERG VAN DEN, R.; HAENEN, G.R.M.M.; BERG VAN DEN, H.; VIJGH VAN DER, W.; BAST, A. The predictive value of the antioxidant capacity of structurally related flavonoids using the Trolox equivalent antioxidant capacity (TEAC) assay. Food Chemistry, Columbus, v. 70, n. 3, p. 391-395, 2000.

CAI, Y.; LUO, Q.; SUN, M.; CORKE, H. Antioxidant activity and phenolic compounds of 112 traditional Chinese medicinal plants associated with anticancer. Life Sciences, Stockholm, v. 74, n. 17, p. 2157, 2004.

CÄMMERER, B.; KROH, L.W. Antioxidant activity of coffee brews. European Food Research and Technology, Heidelberg, v. 223, n. 4, p. 469-474, 2006.

CHUN, O.K.; KIM, D.O.; MOON, H.Y.; KANG, H.G.; LEE, C.Y. Superoxide radical Scavenging activity of the major polyphenols in fresh plums. Journal Agricultural and Food Chemistry, Washington, v. 51, n. 27, p. 7240, 2003.

CLAY, J.W.; SAMAPAIO, P.T.B.; CLEMENT, C.R. Biodiversidade amazônica: exemplos e estratégias. Manaus: programa de desenvolvimento empresarial e tecnológico, SEBRAE, 1999. p.409.

FENNEMA, O. R. Food Chemistry. 3.ed. New York: Marcel Dekker, 1996.

GIL, M.I.; TOMÁS-BARBERÁN, F.A.; HESSPIERCE, B.; KADER, A.A. Antioxidant capacities, phenolic compounds, carotenoids, and vitamin $\mathrm{c}$ contents of nectarine, peach, and plum cultivars from California. Journal Agricultural and Food Chemistry, Washington, v.50, n.17, p.4976, 2002.

HALLIWELL, B. Antioxidants in human health and disease. Annual Review of Nutrition, Gainesville, v.16, p.33-50, 1996.

HUANG, D.; OU, B.; PRIOR, R.L. The chemistry behind antioxidant capacity assays. Journal Agricultural and Food Chemistry, Washington, v.53, p.1841-1856, 2005.
INSTITUTO ADOLFO LUTZ. Normas analíticas do IAL.: métodos químicos e físicos para análise de alimentos. 5.ed. São Paulo, 2008. 1020 p.

KIM, D.; JEONG, S.; LEE, C. Y. Antioxidant capacity of phenolic phytochemicals from various cultivars of plums. Food Chemistry, Columbus, v.81, p.321-326, 2003.

KITTS, D.D. Bioactive substances in food: identification and potential uses. Canadian Journal of the Physiology and Pharmacology, Ottawa, v. 72, n. 4, p.23-434, 1994.

LABUZA, T.P. Kinetics of lipid oxidation in foods Critical Review in Food Technology, Cleveland, v.2, n.10, p.355-395, 1971.

LAWLESS, H.T.; HEYMANN, H. Sensory evaluation of food. New York: Chapman \& Hall, 1998. 819 p.

LUXIMON-RAMMA, A.; BAHORUN, T.; CROZIER, A.. Antioxidant actions and phenolic and vitamin contents of common Mauritian exotic fruits. Journal of Science Food Agriculture, Chichester, v. 83, n. 5 , p.496-502, 2003.

OLIVEIRA, E.B.; BASTOS, M.S.R.; FEITOSA, T.; BRANCO, M.A.A.C.; SILVA, M.G.G. Avaliação de parâmetros de qualidade físico-químicos de polpas congeladas de acerola, cajá e caju. Ciência e Tecnologia de Alimentos, Campinas, v.19, n.3, p.326-332, 1999.

PRIOR, R.L.; WU, X.; SCHAICH, K. Standardized methods for the determination of antioxidant capacity and phenolics in foods and dietary supplements. Journal of Agricultural and Food Chemistry, Washington, v.53, p. 4290-4303, 2005.

ROMERO, T. Amazônia em pé vale muito mais. Disponível em: <www.ambienteemfoco.com. br/?p=5096>. Acesso em: jul. 2009.

RUFINO, M.S.M.; FERNANDES, F.A.N.; ALVES, R.E.; BRITO, E.S. Free radical-scavenging behaviour of some north-east Brazilian fruits in a DPPH system. Food Chemistry, Columbus, v.114, n.2, p.693-695, 2009. 
SÁNCHEZ-GONZÁLEZ, I.; JIMÉNEZ-ESCRIG, A.; SAURA-CALIZTO, F. In vitro antioxidant activity of brewed using different procedures (Italian, espresso and filter). Food Chemistry, Columbus, v.90, p.133-139, 2005.

SILVA, E.M.; SOUZA, J.N.S.; ROGEZ, H.; REES, J.F.; LARONDELLE, Y. Antioxidant activities and polyphenolic contents of fifteen selected plant species from the Amazonian region. Food Chemistry, Columbus, v.101, n.3, p.1012, 2007.

SILVA, P.S.L.; SÁ, W.R.; MARIGUELE, K.H.; BARBOSA, A.P.R.; OLIVEIRA, O.F. Distribuição do teor de sólidos solúveis totais em frutos de algumas espécies de clima temperado. Revista Caatinga, Mossoró, v. 15, n. 1/2, p.19-23, 2002.

SOARES, S. E. Ácidos fenólicos como antioxidantes. Revista de Nutrição, Campinas, v.15, n.1, p.71-81, 2002.
SOUSA, C.M.M.; ROCHA E SILVA, H.; VIEIRAJR, G.M.; AYRES, M.C. C.; COSTA, C.L.S.; ARAÚJO, D.S.; CAVALCANTE, L.C.D.; BARROS, E.D.S.; ARAÚJO, P.B.M.; BRANDÃO, M.S.; CHAVES, M.H. Fenóis totais e atividade antioxidante de cinco plantas medicinais. Química Nova, São Paulo, v. 30, n.2, p.351-355, 2007

SOUZA, V.A.B.; ARAÚJO, E.C.E.; VASCONCELOS, L.F.L.; LIMA, P.S.C. Variabilidade de características físicas e químicas de frutos germoplasma de bacuri da região meio-norte do Brasil. Revista Brasileira de Fruticultura, Jaboticabal, v.23, n.3, p.677-683, 2001.

STATSOFT. STATISTICA for Windows: computer program manual. Versão 6.0. Tulsa, 2001.

YAHIA, E. M. The Contribution of Fruit and Vegetable Consumption to Human Health. In: ROSA, L.A.; ALVAREZ-PARRILLA, E.; GONZALEZAGUILARA; G.A. Fruit and vegetable phytochemicals: chemistry, nutritional value and stability. Hoboken: Wiley-Blackwell, 2010. p. 3-51. 